\title{
D-branes with Lorentzian signature in the Nappi-Witten model
}

\author{
R. Hernández, G. Horcajada and F. Ruiz Ruiz \\ Departamento de Física Teórica I, Universidad Complutense de Madrid, \\ 28040 Madrid, Spain \\ E-mail: rafael.hernandez@fis.ucm.es, ghr@fis.ucm.es, \\ ferruiz@fis.ucm.es
}

AbStRaCt: Lorentzian signature D-branes of all dimensions for the Nappi-Witten string are constructed. This is done by rewriting the gluing condition $J_{+}=F J_{-}$for the model chiral currents on the brane as a well posed first order differential problem and by solving it for Lie algebra isometries $F$ other than Lie algebra automorphisms. By construction, these D-branes are not twined conjugacy classes. Metrically degenerate D-branes are also obtained.

Keywords: D-branes, Penrose limit and pp-wave background

ARXIV EPRINT: 1104.4730 


\section{Contents}

$\begin{array}{llr}1 & \text { Introduction } & 1\end{array}$

2 Characterization of D-branes in WZW models 2

3 The Nappi-Witten model: a brief review $\quad 6$

4 Filling D-branes and D-strings from general isometries 9

4.1 Case $F=-R_{\text {inner }}^{\Omega} \quad 9$

$\begin{array}{lll}4.2 & \text { Case } F=-R_{\text {outer }}^{\Omega} & 11\end{array}$

$5 \quad$ D2 and D0-branes with Lorentzian signature 13

6 Comparison with the sigma-model approach $\quad 15$

$\begin{array}{llr}7 & \text { Outlook } & 18\end{array}$

A Alternative computation of $\operatorname{rank}\left[\operatorname{Ad}_{g^{-1}}\left(-R_{ \pm}^{\Omega}\right)-1\right] \quad 18$

\section{Introduction}

The approach to understanding D-branes and their properties in terms of the open strings attached to their worldvolumes has provided a new look on D-branes. One of the most remarkable and influential results along this line is the observation that, for flat spacetime and a globally defined constant two-form $B$, the D-brane world volume becomes noncommutative upon quantization [1-4]. In particular, if $B$ is of magnetic type, space directions do not commute [1-3], whereas if $B$ is of electric type, it is the time direction that does not commute with the space directions [4]. Since the three-form $H$ vanishes for constant $B$, the field equations for the string are the same as for $B=0$. The boundary conditions, however, change, since they involve the field $B$; the annhilation and creation parts of every string mode get coupled and this coupling leads to noncommutativity upon quantization. Noncommutativity should then be a general feature for D-branes in curved backgrounds.

Two important examples of the latter are provided by (i) the family of $p p$-wave geometries $[5,6]$ with also a globally defined constant $B$ that describe the Penrose limits of $\operatorname{AdS}_{n} \times \mathrm{S}^{m}$ and $\mathrm{dS}_{n} \times \mathrm{S}^{m}$, and (ii) an $\mathrm{S}^{3}$ background with nonzero $H[7,8]$. In the first case, the three-form $H$ vanishes and noncommutativity at the string endpoints can be established through canonical quantization. In the second case, canonical quantization is not adequate and noncommutativity is proved by resorting to the SU(2) WZW formulation of the string background.

More generally, since WZW models are the building blocks of many string backgrounds, one expects to learn about D-branes and their noncommutative field theories by looking 
at open strings on group manifolds. This entails as a first step the characterization of D-branes in WZW models [9-18]. Such characterization is well understood in some cases. In particular, it is known that the metrically nondegenerate $R$-twined conjugacy classes of a WZW group manifold are D-branes for all Lie algebra metric-preserving automorphisms $R$. These twined conjugacy classes are obtained as the solutions to a gluing condition $J_{+}=R J_{-}$that matches the chiral currents $J_{+}$and $J_{-}$of the model at the D-brane. Very little is known, however, if in the gluing condition, instead of a Lie algebra automorphism, an arbitrary isometry $F$ of the Lie algebra metric is considered [18]. This is due to the fact that involutivity (required for the solution to the gluing condition to define a submanifold) holds trivially for Lie algebra automorphisms, whereas for general isometries it usually does not. In this latter case, involutivity often requires to consider isometries $F(g)$ that depend on the group point $g$ at which the gluing condition must be solved.

The Nappi-Witten model [19] is a WZW model describing a four-dimensional noncompact string background. The twined conjugacy classes of its group manifold are well understood [14]. They provide two-dimensional Euclidean D-branes for metric-preserving inner automorphisms and three-dimensional Lorentzian D-branes for metric-preserving outer automorphisms. The purpose of this paper is to go beyond and construct Lorentzian D-branes of dimension one, two, three and four by solving the gluing condition for isometries other than Lie algebra automorphisms. Our motivation aims to constructing noncommutative field theories on noncompact curved backgrounds.

In this paper we apply geometric characterization of D-branes in nonsemisimple Lie groups along the lines of ref. [18] to the Nappi-Witten model. There are other ways to approach the study of D-branes in WZW models. In particular, the so called algebraic program, that uses boundary conformal theory. See e.g. ref. [20] and references therein for compact string backgrounds, ref. [21] for noncompact ones and ref. [22] for their use in the Nappi-Witten model.

The paper is organized as follows. In section 2, we review the semiclassical characterization of D-branes in a WZW model. The material presented there can be found elsewhere [11-13], though it emphasizes some points [18] concerning the rôle of Frobenius theorem and involutivity that have gone somewhat unnoticed in the literature. Section 3 contains a brief account of the Nappi-Witten model, including a complete characterization of its Lie algebra isometries. Sections 4 and 5 are dedicated to constructing the D-branes of interest. In particular, worldvolume filling D-branes, metrically degenerate D2-branes and Lorentzian D1-branes are presented in section 4, whereas Lorentzian D2 and D0-branes are exhibited in section 5. The subject of section 6 is to recover the boundary conditions for the string coordinates from the gluing condition for the chiral currents. Finally, section 7 collects our conclusions.

\section{Characterization of D-branes in WZW models}

Consider a Lie algebra $\mathfrak{g}$ of dimension d over $\mathbf{R}$ and an invariant Lie algebra metric $\Omega$ defined on it. In a basis $\left\{T_{a}\right\}$, with commutation relations

$$
\left[T_{a}, T_{b}\right]=f_{a b}{ }^{c} T_{c},
$$


the metric components $\Omega_{a b}=\Omega\left(T_{a}, T_{b}\right)$ satisfy

$$
f_{a b}{ }^{d} \Omega_{d c}=\Omega_{a d} f_{b c}{ }^{d} .
$$

Here we will be interested in isometries of $\Omega$. An isometry of $\Omega$ is a linear map $F$ from $\mathfrak{g}$ to $\mathfrak{g}$ such that $\Omega\left(F T_{a}, F T_{b}\right)=\Omega\left(T_{a}, T_{b}\right)$. Writing the action of $F$ on a generator $T_{a}$ as $F\left(T_{a}\right)=T_{b} F_{a}^{b}$, with $F_{a}^{b}$ taking values in $\mathbf{R}$, the isometry condition becomes ${ }^{1}$

$$
\Omega_{a b}=F_{a}^{c} \Omega_{c d} F_{b}^{d} \Leftrightarrow F^{\mathrm{T}} \Omega F=\Omega .
$$

The isometries of $\Omega$ form a subgroup $I s o(\Omega)$ of the general linear group $G \ell(\mathrm{d}, \mathbf{R})$. Our conventions for matrix notation is that the first index, from left to right, labels rows, and the second one labels columns.

The pair $(\mathfrak{g}, \Omega)$ defines a WZW model described by mappings $g$ from the string worldsheet $\Sigma$ to the group manifold $G$ obtained from $\mathfrak{g}$ through exponentiation. If $G$ is locally parameterized by the string coordinates $X^{\mu}(\tau, \sigma)$, the left-invariant $e^{a}{ }_{\mu}$ and right-invariant $\bar{e}^{a}{ }_{\mu}$ vielbeins at $g(X)$ are

$$
g^{-1} d g=T_{a} e^{a}{ }_{\mu} d X^{\mu}, \quad d g g^{-1}=T_{a} \bar{e}_{\mu}^{a} d X^{\mu} .
$$

The adjoint action of the group $G$ on the algebra $\mathfrak{g}$ is

$$
\operatorname{Ad}_{g}\left(T_{a}\right)=g T_{a} g^{-1}=T_{b} \bar{e}^{b}{ }_{\mu}\left(e^{-1}\right)^{\mu}{ }_{a} \quad \Leftrightarrow \quad \operatorname{Ad}_{g}=\bar{e} e^{-1},
$$

where $\left(e^{-1}\right)^{\mu}{ }_{a}$ is the inverse of $e^{a}{ }_{\mu}$, defined by $\left(e^{-1}\right)^{\mu}{ }_{a} e^{b}{ }_{\mu}=\delta_{a}{ }^{b}$. The spacetime metric $G_{\mu \nu}$ and the three-form $H_{\mu \nu \lambda}$ specifying the string background are given in terms of $\Omega$ by

$$
\begin{aligned}
G_{\mu \nu} & =\Omega\left(g^{-1} \partial_{\mu} g, g^{-1} \partial_{\nu} g\right) \\
H_{\mu \nu \lambda} & =\Omega\left(\left[g^{-1} \partial_{\mu} g, g^{-1} \partial_{\nu} g\right], g^{-1} \partial_{\lambda} g\right) .
\end{aligned}
$$

In world sheet coordinates $\sigma^{ \pm}=\tau \pm \sigma$, the chiral currents of the model read

$$
J_{-}\left(\sigma^{-}\right)=g^{-1} \partial_{-} g, \quad J_{+}\left(\sigma^{+}\right)=-\partial_{+} g g^{-1}
$$

and satisfy $\partial_{+} J_{-}=\partial_{-} J_{+}=0$.

A Dp-brane is a $(p+1)$-dimensional submanifold $N$ of $G$ on which an open string may end. Points in $N$ can be parameterized by the string endpoints coordinates $x^{\mu}(\tau)=\left.X^{\mu}(\tau, \sigma)\right|_{\partial \Sigma}$, so we will write $g(x)$. The D-brane can be specified $[9,12,13,18]$ by

(i) An isometry $F$ of $\Omega$, that in general may depend on $g$, and a condition

$$
J_{+}=F(g) J_{-} \quad \text { at } \quad \partial \Sigma .
$$

This condition must define $i=1, \ldots, p+1$ integrable vector fields $k_{i}(x)=k^{\mu}{ }_{i}(x) \partial_{\mu}$ that characterize the tangent bundle of the submanifold $N$. The fields $k_{i}(x)$ must define a basis of $T_{g} N$ for all $g(x)$ in $N$. In what follows we will denote by $\alpha^{i}$ the local coordinates along the directions defined by $k_{i}$, that is, $k_{i}=\partial / \partial \alpha^{i}$.

\footnotetext{
${ }^{1}$ In matrix notation, in $F_{b}^{a}$ the index $a$ specifies the row and the index $b$ the column.
} 
(ii) A two-form $\omega$ defined on $N$, with components $\omega_{i j}=\omega\left(k_{i}, k_{j}\right)$ satisfying the following two requirements. Firstly, eq. (2.7) must reproduce the usual boundary conditions of the sigma model formulation, which in the presence of a D-brane read $[9,18]$

$$
\left.\left(k_{i}^{\mu} G_{\mu \nu} \partial_{\sigma} X^{\nu}-\omega_{i j} \partial_{\tau} \alpha^{j}\right)\right|_{\partial \Sigma}=0 \quad i=1, \ldots, p+1 .
$$

And secondly, $d \omega=\left.H\right|_{N}$. Note that the variations of the D-brane coordinates $\alpha^{i}$ and the string endpoints coordinates $x^{\mu}$ with $\tau$ are related by $\partial_{\tau} \alpha^{i} k^{\mu}{ }_{i}=\partial_{\tau} x^{\mu}$.

We remark that, from the viewpoint of the sigma model, the boundary conditions take the form (2.8). These are the equations that must be recovered from eq. (2.7). To avoid confusion, eq. (2.7) is called gluing condition.

Writing the chiral currents as $J_{-}=T_{a} e^{a}{ }_{\mu} \partial_{-} X^{\mu}$ and $J_{+}=-T_{a} \bar{e}_{\mu}^{a} \partial_{+} X^{\mu}$, multiplying from the left with the right-invariant inverse vielbein, and using world sheet coordinates $\tau$ and $\sigma$, condition $(2.7)$ is written as

$$
\left.(\mathcal{F}-1) \partial_{\tau} X\right|_{\partial \Sigma}=\left.(\mathcal{F}+1) \partial_{\sigma} X\right|_{\partial \Sigma}
$$

Here $\mathcal{F}$ stands for

$$
\mathcal{F}^{\mu}{ }_{\nu}=-\left(\bar{e}^{-1}\right)^{\mu}{ }_{a} F^{a}{ }_{b} e^{b}{ }_{\nu} \quad \Leftrightarrow \quad \mathcal{F}(x)=-\bar{e}^{-1} F(g) e
$$

and is called matrix of boundary conditions. $\mathcal{F}$ is only defined at $\partial \Sigma$ and depends on $x^{\mu}$ through the vielbeins $e(x)$ and $\bar{e}^{-1}(x)$ and the isometry $F(g(x))$.

For any $g$ in $G$, the only motions compatible with conditions (2.9) are along the curves tangent to the vector fields $[12,13,18]$

$$
t_{U}(g)=F U g-g U, \quad U \in \mathfrak{g} .
$$

Since $U=U^{a} T_{a}$ for all $U$ in $\mathfrak{g}$ and $\left\{T_{a}\right\}$ is a basis, it is enough to consider the fields

$$
t_{a}(g)=F T_{a} g-g T_{a} .
$$

At every $g$, the fields $t_{a}(g)$ define a space of tangent directions

$$
\Pi_{g}=\operatorname{Span}\left\{t_{a}(g)\right\}
$$

contained in the tangent space $T_{g} G$ at $g$ to the whole manifold $G$. If the tangent planes $\Pi_{g}$ have dimension $p+1$ for all $g$ in $G$, their collection defines a $(p+1)$-dimensional distribution on $G$,

$$
\Pi=\left\{\Pi_{g}: \operatorname{dim} \Pi_{g}=p+1, g \in G\right\} .
$$

Multiplication of $t_{a}(g)$ from the left with $g^{-1}$ gives $g^{-1} t_{a}=\operatorname{Ad}_{g^{-1}} F T_{a}-T_{a}$. The planes $\Pi_{g}$ have dimension $p+1$ for all $g$ if and only if $\operatorname{Ad}_{g^{-1}} F-1$ has rank $p+1$ for all $g$. It may occur that $\Pi_{g}$ does not have constant dimension over $G$, so that $\Pi$ is not a distribution on $G$. In this case, since the open string endpoints flow along the integral curves of $t_{a}$, it is enough to have a distribution

$$
\Pi^{\prime}=\left\{\Pi_{g}: \operatorname{dim} \Pi_{g}=p+1, g \in G^{\prime}\right\}
$$


on a submanifold $G^{\prime}$ of $G$, provided $G^{\prime}$ contains all such curves. See ref. [18] for details and sections 4 and 5 for examples.

For $\Pi_{g}$ to be at all $g$ in $G^{\prime}$, not just a tangent plane, but the tangent space to a submanifold $N_{p+1}$ of $G^{\prime}$, the distribution $\Pi^{\prime}$ must be integrable. According to Frobenius theorem, $\Pi^{\prime}$ is integrable if and only if the vector fields $t_{a}$ are involutive. That is, if and only if the commutator $\left[t_{a}, t_{b}\right]$ of any two fields $t_{a}$ and $t_{b}$ taking values in $\Pi^{\prime}$ also takes values in $\Pi^{\prime}$. This amounts to the existence of functions $c_{a b}{ }^{c}(g)$ such that

$$
\left[t_{a}(g), t_{b}(g)\right]=c_{a b}^{c}(g) t_{c}(g)
$$

for all $g$ in $G^{\prime}$. The distribution $\Pi^{\prime}$ is the tangent bundle of $N_{p+1}$.

The field $t_{a}(g)$ is the sum of a right-invariant vector field $X_{\mathrm{R}} g$, with $X_{\mathrm{R}}=F T_{a}$, and a left-invariant one $g X_{\mathrm{L}}$, with $X_{\mathrm{L}}=-T_{a}$. Its action on a differentiable function $f$ defined on $G$ is easily computed from the actions of $X_{\mathrm{R}} g$ and $g X_{\mathrm{L}}$, given by

$$
X_{\mathrm{R}} g(f(g))=\left.\frac{d}{d t} f\left(e^{t X_{\mathrm{R}}} g\right)\right|_{t=0}, \quad g X_{\mathrm{L}}(f(g))=\left.\frac{d}{d t} f\left(g e^{t X_{\mathrm{L}}}\right)\right|_{t=0} .
$$

If $g(x)$ is parameterized by coordinates $x^{\mu}$, the components of $X_{\mathrm{R}} g$ and $g X_{\mathrm{L}}$ are

$$
\begin{gathered}
X_{\mathrm{R}} g=X_{\mathrm{R}}^{a} T_{a} g=X_{\mathrm{R}}^{a}\left(\bar{e}^{-1}\right)^{\mu}{ }_{a} \partial_{\mu} \\
g X_{\mathrm{L}}=X_{\mathrm{L}}^{a} g T_{a}=X_{\mathrm{L}}^{a}\left(e^{-1}\right)^{\mu}{ }_{a} \partial_{\mu} .
\end{gathered}
$$

This gives

$$
t_{a}(x)=F T_{a} g-g T_{a}=\left[\left(\bar{e}^{-1}\right)^{\mu}{ }_{b} F^{b}{ }_{a}-\left(e^{-1}\right)^{\mu}{ }_{a}\right] \partial_{\mu}=t^{\mu}{ }_{a}(x) \partial_{\mu} .
$$

The rank of the matrix $t_{a}^{\mu}(x)$ at $g(x)$ is the dimension of $\Pi_{g}$. Note that the $a$-th column of this matrix is formed by the components of the tangent vector $t_{a}(x)$.

Let us restrict our attention for the time being to isometries $F$ that are constant over $G$. In this case, equation (2.12) takes the simpler form [18]

$$
-\left[F T_{a}, F T_{b}\right] g+g\left[T_{a}, T_{b}\right]=c_{a b}^{c}(g)\left(F T_{c} g-g T_{c}\right) .
$$

A solution to this equation is provided by $F=R^{\Omega}$ and $c_{a b}{ }^{c}(g)=-f_{a b}{ }^{c}$, with $R^{\Omega}$ a Lie algebra automorphism satisfying eq. (2.3) and $f_{a b}{ }^{c}$ the Lie algebra structure constants. This is trivial since, by definition, Lie algebra automorphisms $R$ satisfy

$$
R\left[T_{a}, T_{b}\right]=\left[R T_{a}, R T_{b}\right]
$$

The restriction to automorphisms $R^{\Omega}$ complying with eq. (2.3) comes from the observation that for a general Lie algebra not all automorphisms $R$ are isometries. Automorphisms fulfilling (2.3) condition are called $\Omega$-preserving. The vector fields $t_{a}=R^{\Omega} T_{a} g-g T_{a}$ are very easy to integrate and give for the submanifold $N$ the $R^{\Omega}$-twined conjugacy classes of the group $G[10,12,13]$,

$$
N=\mathcal{C}\left(R, g_{0}\right)=\left\{e^{R^{\Omega} V} g_{0} e^{-V}: V \in \mathfrak{g}\right\}
$$


where $g_{0}$ is an arbitrary group element that accounts for the integration constants.

There are suggestions $[11,12,24]$ that $F=-R^{\Omega}$, with $R^{\Omega}$ an $\Omega$-preserving constant automorphism, may solve involutivity and, hence, may lead to D-branes. For semisimple Lie algebras, however, it has been proved [18] that this is not the case. In the following sections we examine this problem for the Nappi-Witten model, a typical example of nonsemisimple WZW model. We find that $F=-R^{\Omega}$ does not define D-branes if $R^{\Omega}$ is constant, but it does if $R^{\Omega}$ is conveniently taken to depend on $g$. The choice of the $g$-dependence of $R^{\Omega}(g)$ is indicated by the requirement of the constancy of the rank of the matrix $t_{a}^{\mu}(x)$ In sections 4 to 6 several examples are presented.

\section{The Nappi-Witten model: a brief review}

The Nappi-Witten model [19] is constructed upon a nonsemisimple Lie algebra $\mathfrak{g}_{\mathrm{Nw}}$ whose exponentiation gives a group manifold $G_{\mathrm{NW}}$ describing a nontrivial four-dimensional string background of $p p$-type. The algebra has dimension four and generators $\left\{P_{1}, P_{2}, J, T\right\}$ with commutation relations

$$
\left[J, P_{M}\right]=\epsilon_{M N} P_{N}, \quad\left[P_{M}, P_{N}\right]=\epsilon_{M N} T, \quad\left[T, P_{i}\right]=[T, J]=0, \quad M, N=1,2 .
$$

It is the central extension of the Euclidean algebra in two dimensions, $T$ being the central charge. We will use the labeling

$$
T_{1}=P_{1}, \quad T_{2}=P_{2}, \quad T_{3}=J, \quad T_{4}=T,
$$

so that

$$
f_{12}^{4}=f_{31}^{2}=f_{23}{ }^{1}=1
$$

The most general invariant metric $\Omega$ can be found by solving eqs. (2.2). It reads [19]

$$
\Omega=k\left(\begin{array}{llll}
1 & 0 & 0 & 0 \\
0 & 1 & 0 & 0 \\
0 & 0 & b & 1 \\
0 & 0 & 1 & 0
\end{array}\right),
$$

where $k$ and $b$ are arbitrary real parameters. The parameter $k$ can be absorbed in the coupling constant in front of the classical WZW action, so that it can be set equal to one without loss of generality. As concerns $b$, it can be set to zero by the following redefinition of the Lie algebra generators:

$$
P_{M}^{\prime}=P_{M}, \quad J^{\prime}=J-\frac{b}{2} T, \quad T^{\prime}=T .
$$

Indeed, under such transformations, the Lie algebra commutators (3.1) remain unchanged and the metric $\Omega$ takes the form in (3.3) with $b=0$. We thus set $k=1$ and $b=0$ without loss of generality. 
Lie algebra isometries. The isometries $F$ of $\Omega$ are the solutions to equation (2.3). To find them, it is most convenient to write $\Omega$ in eq. (3.3) as $\Omega=M^{\mathrm{T}} \eta M$, with $M$ the matrix

$$
M=\left(\begin{array}{cccc}
1 & 0 & 0 & 0 \\
0 & 1 & 0 & 0 \\
0 & 0 & \frac{\sqrt{2}}{2} & \frac{\sqrt{2}}{2} \\
0 & 0 & \frac{\sqrt{2}}{2} & -\frac{\sqrt{2}}{2}
\end{array}\right)
$$

and $\eta=\operatorname{diag}(+,+,+,-)$. Eq. (2.3) then becomes

$$
\left(M F M^{-1}\right)^{\mathrm{T}} \eta\left(M F M^{-1}\right)=\eta .
$$

This is solved by $M F M^{-1}$ an arbitrary element of $O(3,1)$, so the isometry group is

$$
\operatorname{Iso}(\Omega)=\left\{M^{-1} \Lambda M: \Lambda \in O(3,1)\right\} .
$$

Note that $M$ is not an isometry.

Lie algebra automorphisms. The automorphisms of the Nappi-Witten algebra can be found by solving eqs. (2.17). Using the structure constants $f_{a b}{ }^{c}$ in eq. (3.2), it is straightforward to see that eqs. (2.17) only have two solutions, $R_{+}$and $R_{-}$, given by

$$
R_{ \pm}\left(\rho_{0}, \rho, \phi, \theta\right)=\left(\begin{array}{cccc}
\rho_{0} \cos \phi & \mp \rho_{0} \sin \phi & \mp \frac{\rho}{\rho_{0}} \cos \theta & 0 \\
\rho_{0} \sin \phi & \pm \rho_{0} \cos \phi & -\frac{\rho}{\rho_{0}} \sin \theta & 0 \\
0 & 0 & \pm 1 & 0 \\
\rho \cos (\theta \mp \phi) & \rho \sin (\theta \mp \phi) & \zeta & \pm \rho_{0}^{2}
\end{array}\right) .
$$

The parameters $\rho_{0}, \rho, \phi, \theta$ and $\zeta$ can take any values on the ranges

$$
\rho_{0}>0, \quad \rho \geq 0, \quad 0 \leq \phi, \theta<2 \pi, \quad-\infty<\zeta<\infty .
$$

As is well known, the automorphisms $R_{-}$and $R_{+}$form a group, $\operatorname{Aut}\left(\mathfrak{g}_{\mathrm{NW}}\right)$. There are a few observations concerning automorphisms and isometries that we find relevant:

- $\operatorname{Aut}\left(\mathfrak{g}_{\mathrm{NW}}\right)$ is not $O(3,1)$ nor a subgroup of it. This would require $R_{ \pm}$to satisfy $R_{ \pm}^{\mathrm{T}} \eta R_{ \pm}=\eta$, but this only occurs for $\rho_{0}=1, \zeta=\rho=0$.

- Not every isometry is a Lie algebra automorphism. Take for example

$$
F_{0}=M^{-1} \eta M=\left(\begin{array}{llll}
1 & 0 & 0 & 0 \\
0 & 1 & 0 & 0 \\
0 & 0 & 0 & 1 \\
0 & 0 & 1 & 0
\end{array}\right)
$$

By construction, $F_{0}$ is an isometry but does not have the form (3.4), hence is not an automorphism. $^{2}$

\footnotetext{
${ }^{2}$ As a matrix $F_{0}$ is equal to $\Omega$, but they have different index structures: $\left(F_{0}\right)^{a}{ }_{b}$ and $\Omega_{a b}$.
} 
- Conversely, not every automorphism is an isometry. For this to be the case, $R_{ \pm}$must satisfy $R_{ \pm}^{\mathrm{T}} \Omega R_{ \pm}=\Omega$. Some algebra shows that this is so if and only if $\rho_{0}=1$ and $\zeta=\mp \rho^{2} / 2$. From now on, we denote by $R_{ \pm}^{\Omega}$ automorphisms of this type,

$$
R_{ \pm}^{\Omega}(\rho, \phi, \theta)=\left(\begin{array}{cccc}
\cos \phi & \mp \sin \phi & \mp \rho \cos \theta & 0 \\
\sin \phi & \pm \cos \phi & -\rho \sin \theta & 0 \\
0 & 0 & \pm 1 & 0 \\
\rho \cos (\theta \mp \phi) & \rho \sin (\theta \mp \phi) & \mp \frac{\rho^{2}}{2} & \pm 1
\end{array}\right)
$$

They form the subgroup $\operatorname{Aut}_{\Omega}\left(\mathfrak{g}_{\mathrm{NW}}\right)$ of $\Omega$-preserving automorphisms.

The spacetime group manifold. A group element $g$ can be parameterized as [19]

$$
g\left(x_{M}, u, v\right)=e^{x_{M} P_{M}} e^{u J} e^{v T}, \quad M=1,2
$$

in terms of real coordinates $x_{M}, u, v$. In this parameterization, the identity element is $e=g(0,0,0)$, while the group law takes the form $g(x) g\left(x^{\prime}\right)=g\left(x^{\prime \prime}\right)$, with

$$
\begin{aligned}
x_{M}^{\prime \prime} & =x_{M}+\cos u x_{M}^{\prime}-\sin u \epsilon_{M K} x_{K}^{\prime} \\
u^{\prime \prime} & =u+u^{\prime} \\
v^{\prime \prime} & =v+v^{\prime}+\frac{1}{2} \cos u \epsilon_{M K} x_{M} x_{K}^{\prime}+\frac{1}{2} \sin u x_{M} x_{M}^{\prime} .
\end{aligned}
$$

The inverse $g^{-1}$ of $g$ reads

$$
g\left(x_{M}, u, v\right)^{-1}=g\left(-\cos u x_{M}-\sin u \epsilon_{M K} x_{K},-u,-v\right) .
$$

The left and right-invariant vielbeins follow easily from their definition (2.4). They read

$$
e_{\mu}^{a}=\left(\begin{array}{cccc}
\cos u & \sin u & 0 & 0 \\
-\sin u & \cos u & 0 & 0 \\
0 & 0 & 1 & 0 \\
\frac{x_{2}}{2} & -\frac{x_{1}}{2} & 0 & 1
\end{array}\right), \quad \bar{e}^{a}{ }_{\mu}=\left(\begin{array}{cccc}
1 & 0 & x_{2} & 0 \\
0 & 1 & -x_{1} & 0 \\
0 & 0 & 1 & 0 \\
-\frac{x_{2}}{2} & \frac{x_{1}}{2} & -\frac{1}{2}\left(x_{1}^{2}+x_{2}^{2}\right) & 1
\end{array}\right) .
$$

Some simple algebra gives then for the adjoint action of the group on the Lie algebra

$$
\operatorname{Ad}_{g}=\bar{e} e^{-1}=\left(\begin{array}{cccc}
\cos u & -\sin u & x_{2} & 0 \\
\sin u & \cos u & -x_{1} & 0 \\
0 & 0 & 1 & 0 \\
x_{1} \sin u-x_{2} \cos u & x_{1} \cos u+x_{2} \sin u & -\frac{1}{2}\left(x_{1}^{2}+x_{2}^{2}\right) & 1
\end{array}\right) .
$$

It follows that $R_{+}^{\Omega} U=\operatorname{Ad}_{h} U$ for all $U$ in the Nappi-Witten algebra, with $h$ a group element with coordinates

$$
x_{1}=\rho \sin \theta, \quad x_{2}=-\rho \cos \theta, \quad u=\phi, \quad v \text { arbitrary } .
$$


This implies that automorphisms of type $R_{+}^{\Omega}$ are inner. As regards automorphisms of type $R_{-}^{\Omega}$, they are outer since there is no group element $h$ such that $R_{-}^{\Omega} U=\operatorname{Ad}_{h} U$ for all $U$.

The spacetime metric and the WZW three-form are given by eqs. (2.5) and (2.6). In the coordinates that we are using they take the form

$$
\begin{aligned}
d s^{2} & =d x_{1}^{2}+d x_{2}^{2}+\left(x_{2} d x_{1}-x_{1} d x_{2}\right) d u+2 d u d v \\
H & =d x_{1} \wedge d x_{2} \wedge d u .
\end{aligned}
$$

\section{Filling D-branes and D-strings from general isometries}

We are interested in finding if isometries of the form $F=-R^{\Omega}$ define D-branes. We will consider both constant and $g$-dependent automorphisms. It is convenient to separately discuss inner and outer automorphisms.

\subsection{Case $F=-R_{\text {inner }}^{\Omega}$}

Using eqs. (3.6) and (2.15), for $F=-R_{+}^{\Omega}$, we obtain the following vector fields $t_{a}$ :

$$
\begin{aligned}
t_{1}(x)= & -(\cos \phi+\cos u) \partial_{1}-(\sin \phi+\sin u) \partial_{2} \\
& +\frac{1}{2}\left[x_{1}(\sin \phi-\sin u)-x_{2}(\cos \phi-\cos u)-2 \rho \cos (\theta-\phi)\right] \partial_{v} \\
t_{2}(x)= & (\sin \phi+\sin u) \partial_{1}-(\cos \phi+\cos u) \partial_{2} \\
& +\frac{1}{2}\left[x_{1}(\cos \phi-\cos u)+x_{2}(\sin \phi-\sin u)-2 \rho \sin (\theta-\phi)\right] \partial_{v} \\
t_{3}(x)= & \left(x_{2}+\rho \cos \theta\right) \partial_{1}-\left(x_{1}-\rho \sin \theta\right) \partial_{2}-2 \partial_{u}-\frac{\rho}{2}\left(x_{1} \sin \theta-x_{2} \cos \theta-\rho\right) \partial_{v} \\
t_{4}(x)= & -2 \partial_{v} .
\end{aligned}
$$

They involve the four derivatives $\partial_{1}, \partial_{2}, \partial_{u}$ and $\partial_{v}$. In particular, $\partial_{u}$ only enters $t_{3}$ with constant coefficient, so the motion defined by $t_{3}$ covers the whole range for $u$. A simple calculation shows that

$$
\operatorname{det}\left(t_{a}^{\mu}\right)=8[1+\cos (\phi-u)] .
$$

Nonexistence of D-branes for constant $\boldsymbol{F}=-\boldsymbol{R}_{+}^{\Omega}$. Let us first consider that $R_{+}^{\Omega}$ does not depend on $g(x)$, so the parameters $\rho, \phi, \theta$ are constant. For points $g(x)$ with $u \neq \phi+(2 n+1) \pi$, the determinant (4.5) does not vanish and the tangent planes

$$
\Pi_{g(x)}=\operatorname{Span}\left\{t_{1}, t_{2}, t_{3}, t_{4}\right\} \text { for } u \neq \phi+(2 n+1) \pi
$$

have dimension four. At points $g(x)$ with $u=\phi+(2 n+1) \pi$, however, the determinant (4.5) vanishes. In a neighborhood of these points the fields $t_{1}$ and $t_{2}$ become

$$
\begin{aligned}
& t_{1}(x)=\left[x_{1} \sin \phi-x_{2} \cos \phi-\rho \cos (\theta-\phi)\right] \partial_{v} \\
& t_{2}(x)=\left[x_{1} \cos \phi+x_{2} \sin \phi-\rho \sin (\theta-\phi)\right] \partial_{v},
\end{aligned}
$$


while $t_{3}$ and $t_{4}$ remain as in (4.3) and (4.4). The fields $t_{1}, t_{2}$ and $t_{4}$ define then the same tangent direction, namely $\partial_{v}$, so the tangent planes are spanned by $t_{3}$ and $t_{4}$,

$$
\Pi_{g(x)}=\operatorname{Span}\left\{t_{3}, t_{4}\right\} \text { for } u=\phi+(2 n+1) \pi,
$$

and have dimension 2. Hence, the dimension of $\Pi_{g(x)}$ is not the same for all $g(x)$ in $G_{\mathrm{NW}}$, the collection of tangent planes $\Pi_{g(x)}$ is not a distribution on $G_{\mathrm{NW}}$ and Frobenius theorem does not apply. The same conclusion can be reached by studying the rank of $\operatorname{Ad}_{g^{-1}} F-1$ (see the appendix).

One may consider the submanifold

$$
G_{\mathrm{NW}}^{\prime}=G_{\mathrm{NW}}-\{g(x): u=\phi+(2 n+1) \pi\}
$$

that results from removing from $G_{\mathrm{NW}}$ the closed set of group elements $g(x)$ with $u=$ $\phi+(2 n+1) \pi$. The collection

$$
\Pi^{\prime}=\left\{\Pi_{g(x)}: g(x) \in G_{\mathrm{NW}}^{\prime}\right\}
$$

is now a distribution of dimension four on $G_{\mathrm{NW}}^{\prime}$. Furthermore, having maximal dimension, it is trivially involutive. The manifold $G_{\mathrm{NW}}^{\prime}$ cannot, however, be accepted as a D-brane. The reason is that it does not contain the integral curves of $t_{3}$, which connects points $g\left(x^{\prime}\right)$ with $u^{\prime} \neq \phi+(2 n+1) \pi$ with points $g(x)$ with $u=\phi+(2 n+1) \pi$ that are not in $G_{\mathrm{NW}}^{\prime}$, thus contradicting the idea that the string endpoints lie on the D-brane. The gluing condition (2.7) does not define then a D-brane for constant $F=-R_{+}^{\Omega}$.

Filling D-branes and D-strings for nonconstant $\boldsymbol{F}=-\boldsymbol{R}_{+}^{\Omega}$. The situation is very different if $R_{+}^{\Omega}$ depends on $g(x)$. Assume that we take

$$
F_{4}=R_{+}^{\Omega}(\rho, \phi, \theta), \quad \phi(u)=u+\phi_{0}, \quad \phi_{0}=\text { const } \neq(2 n+1) \pi .
$$

The matrix $t^{\mu}$ has now nonvanishing determinant for all $g(x)$, so the collection $\Pi$ of all the tangent planes $\Pi_{g(x)}$ is a distribution of dimension four on $G_{\mathrm{NW}}$. Having maximal dimension, $\Pi$ is trivially involutive and is thus the tangent bundle of $G_{\mathrm{NW}}$ itself. In section 6 we show that the gluing condition (2.9) for $F_{4}$ in eq. (4.6) with $\rho$ and $\theta$ constant can be written as a boundary condition (2.8) for a two-form $\omega$ such that $d \omega=H$ on $G_{\mathrm{Nw}}$. The gluing condition for such an $F_{4}$ hence defines a filling D-brane.

Consider now the isometry

$$
F_{2}=R_{+}^{\Omega}(\rho, \phi, \theta), \quad \phi(u)=u-\pi .
$$

The determinant $\operatorname{det}\left(t^{\mu}{ }_{a}\right)$ then vanishes for all $g(x)$. In the neighborhood of any $g(x)$, the fields $t_{1}$ and $t_{2}$ read

$$
\begin{aligned}
& t_{1}(x)=-\left[x_{1} \sin u-x_{2} \cos u-\rho \cos (\theta-u)\right] \partial_{v} \\
& t_{2}(x)=-\left[x_{1} \cos u+x_{2} \sin u-\rho \sin (\theta-u)\right] \partial_{v},
\end{aligned}
$$

while $t_{3}$ and $t_{4}$ remain as in (4.3) and (4.4). The only partial derivative that occurs in $t_{1}, t_{2}$ and $t_{4}$ is $\partial_{v}$, so they define the same tangent direction. The tangent planes $\Pi_{g(x)}$ 
have dimension two for all $g(x)$ and are spanned by $t_{3}$ and $t_{4}$. Their collection $\Pi_{2}$ is hence a distribution of dimension two on $G_{\mathrm{NW}}$ and Frobenius theorem can be used. It is trivial that $\left[t_{3}, t_{4}\right]=0$, so the distribution is integrable. $\Pi_{2}$ defines a family of two-dimensional submanifolds $N_{2}$ whose tangent space at all $g(x)$ is $T_{g(x)} N_{2}=\Pi_{g(x)}$. In section 6 , we show that the gluing condition can be recast as a boundary condition for a two-form $\omega$ defined on $N_{2}$. Such form trivially satisfies $d \omega=\left.H\right|_{N_{2}}$, so the submanifolds $N_{2}$ are D1-branes and provide a foliation of $G_{\mathrm{NW}}$.

Redefining $v \rightarrow v-b u / 2$ and using eq. (3.8), we have that

$$
G\left(t_{3}, t_{3}\right)=-\left[\left(x_{1}-\rho \sin \theta\right)^{2}+\left(x_{2}+\rho \cos \theta\right)^{2}\right]<0, \quad G\left(t_{3}, t_{4}\right)=8>0, \quad G\left(t_{4}, t_{4}\right)=0 .
$$

Every submanifold $N_{2}$ in the family has then Lorentzian signature and is a D-string. If $\alpha^{1}$ and $\alpha^{2}$ parameterize the integral curves of $k_{1}(x)=t_{3}(x)$ and $k_{2}(x)=t_{4}(x)$ in eqs. (4.3), the D-string is formed by points $x^{\mu}\left(\alpha^{1}, \alpha^{2}\right)$ such that

$$
d x^{\mu}=k^{\mu}{ }_{1}(x) d \alpha^{1}+k^{\mu}{ }_{2}(x) d \alpha^{2} .
$$

The induced metric on the D-string takes the form

$$
d s_{2}^{2}=G\left(k_{1}, k_{1}\right)\left(d \alpha^{1}\right)^{2}+G\left(k_{1}, k_{2}\right) d \alpha^{1} d \alpha^{2} .
$$

Assume now that $\rho$ and $\theta$ depend on $x_{1}, x_{2}$ and $u$, but not on $v$. Noting that eqs. (4.8) imply that $x_{1}, x_{2}$ and $u$ only depend on $\alpha^{1}$, we conclude that $G\left(k_{1}, k_{1}\right)$ only depends on $\alpha^{1}$ and thus eq. (4.9) is a $p p$-wave metric in $1+1$ dimensions.

To find the metric coefficient $G\left(k_{1}, k_{1}\right)$ as a function of $\alpha^{1}$, some further assumptions on $\rho$ and $\theta$ are necessary. For example, for $\rho$ and $\theta$ constant, integrating eqs. (4.8), we obtain

$$
\begin{aligned}
x_{1} & =\rho \sin \theta+r_{0} \cos \left(\alpha^{1}+\varphi_{0}\right) \\
x_{2} & =-\rho \cos \theta-r_{0} \sin \left(\alpha^{1}+\varphi_{0}\right) \\
u & =-2 \alpha+u_{0} \\
v & =-2 \beta+\frac{r_{0} \rho}{2} \cos \left(\alpha^{1}+\varphi_{0}+\theta\right)+v_{0},
\end{aligned}
$$

with $r_{0}, \alpha_{0}, u_{0}$ and $v_{0}$ integration constants. The D-string metric is then $-r_{0}^{2}\left(d \alpha^{1}\right)^{2}+8 d \alpha^{1} d \alpha^{2}$. To the best of our knowledge, the family (4.9) of D-strings has gone unnoticed in the literature.

\subsection{Case $F=-R_{\text {outer }}^{\Omega}$}

For $F=-R_{-}^{\Omega}$, there are only three nonzero vector fields $t_{a}$, given by

$$
\begin{aligned}
t_{1}(x)= & -(\cos \phi+\cos u) \partial_{1}-(\sin \phi+\sin u) \partial_{2} \\
& +\frac{1}{2}\left[x_{1}(\sin \phi-\sin u)-x_{2}(\cos \phi-\cos u)-2 \rho \cos (\theta+\phi)\right] \partial_{v} \\
t_{2}(x)= & -(\sin \phi-\sin u) \partial_{1}+(\cos \phi-\cos u) \partial_{2} \\
& -\frac{1}{2}\left[x_{1}(\cos \phi+\cos u)+x_{2}(\sin \phi+\sin u)+2 \rho \sin (\theta+\phi)\right] \partial_{v} \\
t_{3}(x)= & -\left(x_{2}+\rho \cos \theta\right) \partial_{1}+\left(x_{1}+\rho \sin \theta\right) \partial_{2}-\frac{\rho}{2}\left(x_{1} \sin \theta+x_{2} \cos \theta+\rho\right) \partial_{v} .
\end{aligned}
$$


They involve $\partial_{1}, \partial_{2}$ and $\partial_{v}$, but not $\partial_{u}$, hence they define motions that leave $u$ constant. The matrix $t_{a}^{\mu}$ of the coefficients is now $3 \times 3$, with $a=1,2,3$ and $\mu=1,2, v$. A straightforward calculation gives

$$
\operatorname{det}\left(t_{a}^{\mu}\right)=-2\left[k\left(x_{1}, x_{2}\right)\right]^{2},
$$

where $k\left(x_{1}, x_{2}\right)$ is the function of $x_{1}$ and $x_{2}$

$$
k\left(x_{1}, x_{2}\right)=\left(x_{1}+\rho \sin \theta\right) \cos \left(\frac{\phi+u}{2}\right)+\left(x_{2}+\rho \cos \theta\right) \sin \left(\frac{\phi+u}{2}\right) .
$$

Nonexistence of D-branes for constant $\boldsymbol{F}=-\boldsymbol{R}_{-}^{\boldsymbol{\Omega}}$. At points $g(x)$ with $k\left(x_{1}, x_{2}\right) \neq$ 0 , the determinant (4.13) does not vanish and the fields $t_{a}$ define three-dimensional tangent planes $\Pi_{g(x)}$. By contrast, for $g(x)$ with $k\left(x_{1}, x_{2}\right)=0$, the determinant (4.13) vanishes. It is straightforward to see that the rank of the matrix $t_{a}^{\mu}$ is one in this case, so the corresponding tangent planes $\Pi_{g(x)}$ have dimension one. The collection of all the planes $\Pi_{g(x)}$ is not a distribution on $G_{\mathrm{NW}}$ and Frobenius theorem cannot be used. This conclusion can also be reached by studying the rank of $\operatorname{Ad}_{g^{-1}} F-1$ for $F=-R_{-}^{\Omega}$ (see the appendix).

One could think of removing from $G_{\mathrm{NW}}$ the locus of points for which $k\left(x_{1}, x_{2}\right)=0$. The resulting submanifold $G_{\mathrm{NW}}^{\prime}$ then does not include all the points accessible to the string endpoints, since $k\left(x_{1}, x_{2}\right)=0$ can be reached from $k\left(x_{1}, x_{2}\right) \neq 0$ through the motions defined by the fields $t_{a}$. Hence the gluing condition (2.7) does not define a D-brane for constant $F=-R_{-}^{\Omega}$.

D2 and D0-branes for nonconstant $\boldsymbol{F}=-\boldsymbol{R}_{-}^{\Omega}$. Let us take now $\rho, \phi$ and $\theta$ in $R_{-}^{\Omega}(\rho, \phi, \theta)$ functions of $x_{1}$ and $x_{2}$ such that $k\left(x_{1}, x_{2}\right)=k_{0}$, with $k_{0}$ a nonzero constant. The fields $t_{1}, t_{2}$ and $t_{3}$ define then three-dimensional tangent planes $\Pi_{g(x)}$ for all $g(x)$ in $G_{\mathrm{NW}}$, whose collection $\Pi_{3}$ is a three-dimensional distribution on $G_{\mathrm{NW}}$. Alternatively, $\Pi_{3}$ is a threedimensional distribution on any constant $u=u_{0}$ three-plane $N_{u_{0}}=\left\{g(x) \in G_{\mathrm{NW}}: u=u_{0}\right\}$. The distribution $\Pi_{3}$ is trivially involutive and defines the tangent bundle of the three-plane $u=u_{0}$. This plane has two spacelike directions and one lightlike direction, but no timelike direction, so the metric is degenerate. In section 6 , the gluing condition is written as the boundary condition for a two-form $\omega$ defined on the three-plane $u=u_{0}$ such that $d \omega=\left.H\right|_{u_{0}}=0$, thus proving that the planes $u=u_{0}$ are degenerate D2-branes.

We next consider $\rho, \phi$ and $\theta$ functions of $x_{1}$ and $x_{2}$ such that $k\left(x_{1}, x_{2}\right)=0$ for all $x_{1}$ and $x_{2}$. In the neighborhood of any point $g(x)$ in $G_{\mathrm{NW}}$ the vector fields $t_{a}$ in (4.10)-(4.12) take the form

$$
\begin{aligned}
& t_{1}(x)=-(\cos \phi+\cos u)\left(\partial_{1}+\frac{\rho}{2} \cos \theta \partial_{v}\right)-(\sin \phi+\sin u)\left(\partial_{2}-\frac{\rho}{2} \sin \theta \partial_{v}\right) \\
& t_{2}(x)=-(\sin \phi-\sin u)\left(\partial_{1}+\frac{\rho}{2} \cos \theta \partial_{v}\right)+(\cos \phi-\cos u)\left(\partial_{2}-\frac{\rho}{2} \sin \theta \partial_{v}\right) \\
& t_{3}(x)=-\left(x_{2}+\rho \cos \theta\right)\left(\partial_{1}+\frac{\rho}{2} \cos \theta \partial_{v}\right)+\left(x_{1}+\rho \sin \theta\right)\left(\partial_{2}-\frac{\rho}{2} \sin \theta \partial_{v}\right)
\end{aligned}
$$


It is very easy to convince oneself that these vectors define a one-dimensional distribution $\Pi_{1}$ on $G_{\mathrm{NW}}$. Being one-dimensional, $\Pi_{1}$ is trivially involutive. Its integral curves $N_{1}$ are spacelike since

$$
G\left(t_{a}, t_{a}\right)>0 \quad \text { for } \quad t_{a} \neq 0, \quad a=1,2,3 .
$$

To give their explicit form, some further assumptions on the dependence of $\rho, \phi$ and $\theta$ on $x_{1}, x_{2}$ and $u$ must be made. Let us give some examples.

Take $x_{1}+\rho \sin \theta=0$ and $\phi+u=\phi_{0} \neq 2 n \pi$. Condition $k\left(x_{1}, x_{2}\right)=0$ implies $x_{2}+$ $\rho \cos \theta=0$. These three equations define $\rho$ and $\theta$ in terms of $x_{1}$ and $x_{2}$, and $\phi$ in terms of $u$. The fields $t_{1}, t_{2}$ and $t_{3}$ become

$$
t_{1}=-2 \cos \left(u-\frac{\phi_{0}}{2}\right) t_{0}, \quad t_{2}=2 \sin \left(u-\frac{\phi_{0}}{2}\right) t_{0}, \quad t_{3}=0
$$

where $t_{0}$ stands for

$$
t_{0}=\cos \left(\frac{\phi_{0}}{2}\right)\left(\partial_{1}+\frac{\rho}{2} \cos \theta \partial_{v}\right)+\sin \left(\frac{\phi_{0}}{2}\right)\left(\partial_{2}-\frac{\rho}{2} \sin \theta \partial_{v}\right) .
$$

It is clear that $t_{1}$ and $t_{2}$ do not vanish simultaneously and specify the same direction at every $x^{\mu}$. The integral curves are in this case $v=x_{1}^{0} x_{2}-x_{2}^{0} x_{1}+v_{0}$, with $x_{1}^{0}, x_{2}^{0}$ and $v_{0}$ integration constants.

Assume now that $x_{1}+\rho \sin \theta$ and $x_{2}+\rho \cos \theta$ do not vanish simultaneously. The field $t_{3}$ is then nonvanishing and the integral curves are formed by $x^{\mu}(\alpha)$, with $u=u_{0}$ and $x_{1}, x_{2}$ and $v$ the solutions to

$$
\frac{d x_{1}}{d \alpha}=-\left(x_{2}+\rho \cos \theta\right), \quad \frac{d x_{2}}{d \alpha}=x_{1}+\rho \sin \theta, \quad \frac{d v}{d \alpha}=-\frac{\rho}{2}\left(x_{1} \sin \theta+x_{2} \cos \theta+\rho\right),
$$

where $\alpha$ is a parameter along the curve. For $\rho=0$, the integral curves are circles $x_{1}^{2}+x_{2}^{2}=r_{0}^{2}$ of arbitrary radius $r_{0}$ located on any two-plane $\left(u=u_{0}, v=v_{0}\right)$. A simple solution for $\rho \neq 0$ is provided by $\rho=-x_{1} / \sin \theta_{0}$, with $\theta=\theta_{0} \neq n \pi$ constant and $\phi=-u_{0}$. In this case, the integral curves are parabolas $v=-\frac{1}{4} \cot \theta_{0} x_{1}^{2}+v_{0}$ on any two-plane $\left(x_{2}=x_{2}^{0}, u=u_{0}\right)$.

In any case, being one-dimensional, $d \omega=\left.H\right|_{N_{1}}$ is trivially satisfied, and the curves $N_{1}$ are D0-branes.

\section{$5 \quad$ D2 and D0-branes with Lorentzian signature}

In the previous section we have constructed D3 and D1-branes with Lorentzian signature by integrating the gluing condition for some $g$-dependent isometries $F=-R^{\Omega}$. Here we construct D2-branes and D0-branes, also with Lorentzian signature, for $g$-dependent isometries $F \neq \pm R^{\Omega}$.

Since the product of two isometries is an isometry and $F_{0}$ in eq. (3.5) is an isometry, $F=F_{0} R^{\Omega}$, with $R^{\Omega}$ an arbitrary metric-preserving automorphism, is also an isometry. 
Isometries of this type do not have the form $\pm R^{\Omega}$. Let us take for $R^{\Omega}$ an inner automorphism, so that we will be considering $F=F_{0} R_{+}^{\Omega}$. The corresponding vector fields $t_{a}$ are

$$
\begin{aligned}
t_{1}-\rho \cos (\phi-\theta) t_{4}= & (\cos \phi-\cos u) \partial_{1}+(\sin \phi-\sin u) \partial_{2} \\
& +\frac{1}{2}\left[2 \rho \cos (\phi-\theta)-x_{1}(\sin \phi+\sin u)+x_{2}(\cos \phi+\cos u)\right] \partial_{v} \\
t_{2}+\rho \sin (\phi-\theta) t_{4}=- & (\sin \phi-\sin u) \partial_{1}+(\cos \phi-\cos u) \partial_{2} \\
& \quad-\frac{1}{2}\left[2 \rho \sin (\phi-\theta)+x_{1}(\cos \phi+\cos u)+x_{2}(\sin \phi+\sin u)\right] \partial_{v} \\
t_{3}+\frac{1}{2}\left(\rho^{2}+2\right) t_{4}= & -\left(x_{2}+\rho \cos \theta\right) \partial_{1}+\left(x_{1}-\rho \sin \theta\right) \partial_{2}+\frac{\rho}{2}\left(x_{1} \sin \theta-x_{2} \cos \theta-\rho\right) \partial_{v} \\
t_{4}= & -x_{2} \partial_{1}+x_{1} \partial_{2}+\partial_{u}-\partial_{v}
\end{aligned}
$$

From these expressions it follows that $\operatorname{det}\left(t^{\mu}{ }_{a}\right)=0$. This indicates that there are no filling D-branes for the isometry that we are considering. We look for D-branes of lower dimension.

Let us take $\phi(u)=u$ and $\rho=0$. Since the parameter $\theta$ always occurs in $R_{+}^{\Omega}$ through $\rho \sin \theta$ and $\rho \cos \theta$, see eq. (3.6), we can set without loss of generality $\theta=0$. The isometry $F$ then reads

$$
F_{3}(u)=F_{0} R_{+}^{\Omega}(0, u, 0)
$$

and the fields $t_{a}$ become

$$
\begin{aligned}
& t_{1}=\left(-x_{1} \sin u+x_{2} \cos u\right) \partial_{v} \\
& t_{2}=\left(-x_{1} \cos u-x_{2} \sin u\right) \partial_{v} \\
& t_{3}=-\partial_{u}+\partial_{v} \\
& t_{4}=-x_{2} \partial_{1}+x_{1} \partial_{2}+\partial_{u}-\partial_{v} .
\end{aligned}
$$

The rank of the matrix $t_{a}^{\mu}$ of coefficients is now three for $x_{1}^{2}+x_{2}^{2} \neq 0$, and one for $x_{1}=$ $x_{2}=0$. We discuss these two instances separately.

D2-branes. Consider the four-dimensional submanifold

$$
G_{4}=\left\{g(x) \in G_{\mathrm{NW}}: x_{1}^{2}+x_{2}^{2} \neq 0\right\} .
$$

The group elements $g(x)$ that are not in $G_{4}$ have $x_{1}=x_{2}=0$. As both $x_{1}$ and $x_{2}$ approach zero, the coefficients of $\partial_{1}$ and $\partial_{2}$ in eqs. (5.2)-(5.5) vanish, so the fields $t_{a}$ do not connect points in $G_{4}$ with points outside $G_{4}$. In other words, the integral curves of $t_{a}$ stay in $G_{4}$. Furthermore, since the rank of the matrix $t_{a}^{\mu}$ is three for all $g$ in $G_{4}$, the fields $t_{a}$ define a three-dimensional distribution $\Pi_{3}$ on $G_{4}$ formed by the tangent planes $\Pi_{g}=\operatorname{Span}\left\{t_{2}, t_{3}, t_{4}\right\}$. We may alternatively take

$$
\Pi_{g(x)}=\operatorname{Span}\left\{k_{1}:=-x_{2} \partial_{1}+x_{1} \partial_{2}, \quad k_{2}:=\partial_{u}, \quad k_{3}:=\partial_{v}\right\} .
$$

The commutator of any two fields $k_{1}, k_{2}, k_{3}$ vanishes, thus implying that they are involutive. According to Frobenius theorem, $\Pi_{3}$ is the tangent bundle of a family of threedimensional submanifolds $N_{3}$ foliating $G_{4}$. If $\alpha^{1}, \alpha^{2}$ and $\alpha^{3}$ parameterize the integral curves 
of $k_{1}, k_{2}$ and $k_{3}$, a manifold $N_{3}$ in the family is formed by points $x^{\mu}\left(\alpha^{1}, \alpha^{2}, \alpha^{3}\right)$ such that

$$
d x^{\mu}=k^{\mu}{ }_{1} d \alpha^{1}+k_{2}^{\mu} d \alpha^{2}+k^{\mu}{ }_{3} d \alpha^{3} .
$$

Integrating these equations we obtain

$$
N_{3}: \quad \begin{aligned}
& x_{1}=r_{0} \cos \left(\alpha^{1}+\varphi_{0}\right) \\
& x_{2}=r_{0} \sin \left(\alpha^{1}+\varphi_{0}\right)
\end{aligned}, \quad u=\alpha^{2}+u_{0}, \quad v=\alpha^{3}+v_{0}
$$

with $r_{0}>0, \varphi_{0}, u_{0}$ and $v_{0}$ arbitrary integration constants. Note that $r_{0}=0$ corresponds to $x_{1}=x_{2}=0$, which is excluded from $G_{4}$ and will be discussed below. The induced metric on $N_{3}$ is

$$
d s_{3}^{2}=r_{0}^{2} d \alpha^{1}\left(d \alpha^{1}-d \alpha^{2}\right)+2 d \alpha^{2} d \alpha^{3} .
$$

For every $r_{0}^{2}>0$, this is a $p p$-wave in $2+1$ dimensions. In section 6 it is shown that the gluing condition for the isometry $F_{3}$ can be cast as a boundary condition with an admissible two-form $\omega$ defined on $N_{3}$, thus ensuring that $N_{3}$ is a D2-brane.

D0-branes. Let us now consider the two-dimensional submanifold

$$
G_{2}=\left\{g(x) \in G_{\mathrm{NW}}: x_{1}=x_{2}=0\right\} .
$$

For $g$ in $G_{2}$, the fields $t_{1}$ and $t_{2}$ in (5.2) and (5.3) vanish, while $t_{3}$ and $t_{4}$ in (5.4) and (5.5) are proportional to each other and define a one-dimensional distribution $\Pi_{1}$ on $G_{2}$. Having dimension one, $\Pi_{1}$ is trivially involutive. The integral curves of $t_{4}$ have $x_{1}=x_{2}=0$ and $u$ and $v$ such that

$$
\frac{d u}{d \alpha}=1, \quad \frac{d v}{d \alpha}=-1
$$

with $\alpha$ a parameter along the curve. Integration gives $v+u=c_{0}$, with $c_{0}$ an arbitrary integration constant. These curves are timelike since $G\left(t_{4}, t_{4}\right)=-2<0$. Furthermore, the induced metric on them is $d s_{1}^{2}=-2 d \alpha^{2}$. In section 6 , we prove that the gluing condition for $F_{3}$ with $x_{1}=x_{2}=0$ can be written as a boundary condition with $\omega=0$, hence trivially satisfying $d \omega=H$ on $x_{1}=x_{2}=0$. These timelike lines are then D0-branes.

\section{Comparison with the sigma-model approach}

In sections 4 and 5 we have integrated the gluing condition for a variety of isometries. We have anticipated that, in every one of the case considered, the resulting submanifold $N$ was a D-brane since the corresponding gluing condition could be written as a sigma model boundary condition with a two-form $\omega$ defined on $N$ such that $d \omega=\left.H\right|_{N}$. Let us show this here.

We first note that there always exists a two-form $\omega$ defined on $N$ such that any gluing condition can be written as a boundary condition. For all $g$ in $N, \omega$ is specified [18] by its action on tangent vectors $t_{a}=F T_{a} g-g T_{a}$ in $T_{g} N$ as

$$
\omega\left(F T_{a} g-g T_{a}, F T_{b} g-g T_{b}\right)=G\left(F T_{a} g-g T_{a}, F T_{b} g+g T_{b}\right),
$$


where we note the sign change in the second argument on the right hand side. Recall that the linearly independent vector fields $k_{i}(i=1, \ldots, p+1)$ that span $T_{g} N$ are linear combinations of $t_{a}$ and that the components of $\omega$ are $\omega_{i j}=\omega\left(k_{i}, k_{j}\right)$, so that the two form $\omega$ is completely determined by eq. (6.1). A separate issue is whether $\omega$ satisfies $d \omega=\left.H\right|_{N}$. For D-branes of dimension one and two, $d \omega=\left.H\right|_{N}=0$ is trivial. Dimension three and larger must be discussed case by case. We concentrate on these cases.

Filling D-brane. We start with the isometry $F_{4}$ in eq. (4.6). The submanifold $N_{4}$ obtained by integrating the gluing condition was the whole group $G_{\mathrm{NW}}$. Computation of the corresponding matrix $\mathcal{F}_{4}$ in eq. (2.10) and substitution in eq. (2.9) gives after some algebra

$$
\begin{aligned}
\left.\partial_{\sigma} X_{1}\right|_{\partial \Sigma}= & -\tan \left(\frac{\phi_{0}}{2}\right) \partial_{\tau} x_{2}+\frac{1}{2}\left[\left(x_{1}-\rho \sin \theta\right) \tan \left(\frac{\phi_{0}}{2}\right)-x_{2}-\rho \cos \theta\right] \partial_{\tau} u \\
\left.\partial_{\sigma} X_{2}\right|_{\partial \Sigma}= & \tan \left(\frac{\phi_{0}}{2}\right) \partial_{\tau} x_{1}+\frac{1}{2}\left[x_{1}-\rho \sin \theta+\left(x_{2}+\rho \cos \theta\right) \tan \left(\frac{\phi_{0}}{2}\right)\right] \partial_{\tau} u \\
\left.\partial_{\sigma} U\right|_{\partial \Sigma}= & 0 \\
\left.2 \partial_{\sigma} V\right|_{\partial \Sigma}= & {\left[x_{2}+\rho \cos \theta+\rho \sin \theta \tan \left(\frac{\phi_{0}}{2}\right)\right] \partial_{\tau} x_{1}-\left[x_{1}-\rho \sin \theta+\rho \cos \theta \tan \left(\frac{\phi_{0}}{2}\right)\right] \partial_{\tau} x_{2} } \\
& +\frac{1}{2}\left[x_{1}^{2}+x_{2}^{2}-\rho\left(x_{1} \sin \theta-x_{2} \cos \theta\right)+\rho \tan \left(\frac{\phi_{0}}{2}\right)\left(x_{1} \cos \theta+x_{2} \sin \theta\right)\right] \partial_{\tau} u .
\end{aligned}
$$

These are the gluing conditions for the chiral currents written in terms of $\partial_{\tau} x^{\mu}$ and $\left.\partial_{\sigma} X^{\mu}\right|_{\partial \Sigma}$. We want to compare them with the sigma model boundary conditions (2.8).

Since $N_{4}=G_{\mathrm{NW}}$ and $T_{g} G_{\mathrm{NW}}$ is spanned at all $g$ by the four vector fields $k_{i}=\delta_{i}^{\mu} \partial_{\mu}$, the boundary conditions (2.8) can be written as

$$
\left.\left(G_{\mu \nu} \partial_{\sigma} X^{\nu}-\omega_{\mu \nu} \partial_{\tau} x^{\nu}\right)\right|_{\partial \Sigma}=0
$$

Using the expression for the metric $G_{\mu \nu}$ in eq. (3.8) and noting that $\partial_{\tau} x^{\mu}$ are arbitrary, it is a matter of simple algebra to check that the gluing conditions (6.2)-(6.5) take the form of the boundary conditions (6.6) for any two-form $\omega$ with

$$
\begin{aligned}
\omega_{v \mu} & =0 \\
\omega_{12} & =-\tan \left(\frac{\phi_{0}}{2}\right) \\
\omega_{1 u} & =\frac{1}{2}\left[\left(x_{1}-\rho \sin \theta\right) \tan \left(\frac{\phi_{0}}{2}\right)-x_{2}-\rho \cos \theta\right] \\
\omega_{2 u} & =\frac{1}{2}\left[x_{1}-\rho \sin \theta+\left(x_{2}+\rho \cos \theta\right) \tan \left(\frac{\phi_{0}}{2}\right)\right] .
\end{aligned}
$$

It is a question of algebra to check that these equations can as well be obtained by using (6.1). So far no restriction has been placed on $\rho$ and $\theta$ in $F_{4}$. By taking them such that $d \omega=d x_{1} \wedge d x_{2} \wedge d u=H$, we conclude that the gluing condition for $F_{4}$ defines a filling D-brane. The simplest way to accomplish this is to choose $\rho$ and $\theta$ constant. 
It is known [18] that different isometries may define the same submanifold $N$ but not all of them admit a two-form $\omega$ on $N$ such that $d \omega=\left.H\right|_{N}$. Let us illustrate this with the filling D-brane at hand. We start by recalling [18] that, given an isometry $F$, it is always possible to define a new isometry

$$
F^{\prime}=\operatorname{Ad}_{g} F^{-1} \operatorname{Ad}_{g} .
$$

The gluing condition for $F^{\prime}$ is integrable if and only if it is for $F$, in which case they both yield the same submanifold $N$. The gluing condition (2.9) for $F^{\prime}$ reads

$$
\left.\left(\mathcal{F}^{\prime}-1\right) \partial_{\tau} X\right|_{\partial \Sigma}=\left.\left(\mathcal{F}^{\prime}+1\right) \partial_{\sigma} X\right|_{\partial \Sigma}
$$

Noting that $\mathcal{F}^{\prime}=\mathcal{F}^{-1}$ and multiplying from the left with $\mathcal{F}$, it becomes

$$
\left.(\mathcal{F}-1) \partial_{\tau} X\right|_{\partial \Sigma}=-\left.(\mathcal{F}+1) \partial_{\sigma} X\right|_{\partial \Sigma}
$$

This is the same condition as for $F$, except for a negative sign in front of the partial derivatives $\left.\partial_{\sigma} X\right|_{\partial \Sigma}$. We now take $F_{4}$ and consider the corresponding $F_{4}^{\prime}$. The gluing conditions for $F_{4}^{\prime}$ are then as in eqs. (6.2)-(6.5) with a negative sign in front of every $\left.\partial_{\sigma} X\right|_{\partial \Sigma}$. To recover the sigma model boundary condition (6.6), we must take $\omega^{\prime}=-\omega$, with $\omega$ as in eqs. (6.8)-(6.10). This in turn implies that $d \omega^{\prime}=-H$. We conclude that the gluing condition for $F_{4}^{\prime}$, though integrable, does not define a D-brane.

Degenerate D2-branes. In subsection 4.2 , the planes $u=u_{0}$ were obtained upon integration of the gluing condition for an isometry $F=-R_{-}^{\Omega}(\rho, \phi, \theta)$ with parameters $\rho, \phi$ and $\theta$ such that $k\left(x_{1}, x_{2}\right)$ in eq. (4.14) took a constant value $k_{0} \neq 0$ for all $x_{1}$ and $x_{2}$. For simplicity we set $\rho=0$. The condition $k\left(x_{1}, x_{2}\right)=k_{0}$ then reads

$$
x_{1} \cos \left(\frac{\phi+u_{0}}{2}\right)+x_{2} \sin \left(\frac{\phi+u_{0}}{2}\right)=k_{0}
$$

and the isometry $F=-R_{-}^{\Omega}(0, \phi, 0)$ becomes a function of $x_{1}$ and $x_{2}$. Other choices for $\rho$ are treated similarly.

Calculation of the corresponding $\mathcal{F}_{3}$ and substitution in eq. (2.9) provides the following gluing conditions:

$$
\begin{aligned}
0 & =\partial_{\tau} u \\
\partial_{\sigma} X_{1}+\left.\frac{x_{2}}{2} \partial_{\sigma} U\right|_{\partial \Sigma} & =\frac{2}{k_{0}} \sin \left(\frac{\phi+u_{0}}{2}\right) \partial_{\tau} v \\
-\partial_{\sigma} X_{2}+\left.\frac{x_{1}}{2} \partial_{\sigma} U\right|_{\partial \Sigma} & =\frac{2}{k_{0}} \cos \left(\frac{\phi+u_{0}}{2}\right) \partial_{\tau} v \\
-\left.\partial_{\sigma} U\right|_{\partial \Sigma} & =\frac{2}{k_{0}}\left[\sin \left(\frac{\phi+u_{0}}{2}\right) \partial_{\tau} x_{1}-\cos \left(\frac{\phi+u_{0}}{2}\right) \partial_{\tau} x_{2}\right] .
\end{aligned}
$$

It is very easy to check that eqs. (6.14)-(6.16) can be written as the $i=1,2,3$ boundary conditions that result from taking $k_{1}=\partial_{1}, k_{2}=\partial_{2}$ and $k_{3}=\partial_{v}$ in eqs. (2.8) for $\omega$ given by

$$
\omega_{12}=0, \quad \omega_{13}=\frac{2}{k_{0}} \sin \left(\frac{\phi+u_{0}}{2}\right), \quad \omega_{23}=-\frac{2}{k_{0}} \cos \left(\frac{\phi+u_{0}}{2}\right) .
$$


This expression for $\omega$ can also be obtained by taking $F=-R_{-}^{\Omega}(0, \phi, 0)$ in eq. (6.1). Eqs. (6.12) and (6.17) imply that $d \omega=0$, hence $d \omega=\left.H\right|_{u_{0}}=0$.

Lorentzian D2-branes. We close by considering the isometry $F_{3}(u)$ in eq. (5.1). In section 5 we distinguished two cases: $x_{1}^{2}+x_{2}^{2}=r_{0}^{2} \neq 0$ and $x_{1}=x_{2}=0$. In the first one, integration of the gluing condition resulted in the three-dimensional $p p$-wave in eqs. (5.7) and (5.8), whose tangent space is spanned by the vector fields $k_{1}, k_{2}$ and $k_{3}$ in eq. (5.6). It is straightforward to show, either by direct computation or by using eq. (6.1), that the gluing condition for $\mathcal{F}_{3}$ can be recast as boundary conditions with a two-form $\omega$ given in components, by

$$
\omega_{12}=\omega\left(k_{1}, k_{2}\right)=\frac{r_{0}^{2}}{2}-2, \quad \omega_{13}=\omega\left(k_{1}, k_{3}\right)=-2, \quad \omega_{23}=\omega\left(k_{2}, k_{3}\right)=1 .
$$

It is clear that $d \omega=0$. On the other hand, since $x_{1}^{2}+x_{2}^{2}=r_{0}^{2}$ is a nonzero constant, $d x_{1}$ and $d x_{2}$ are not independent and the three-form $H$ vanishes on $N_{3}$. Hence $d \omega=\left.H\right|_{N_{3}}$ is trivially satisfied.

D-strings. The two-form $\omega$ for any 1-dimensional D-brane is trivially zero. Let us for completeness compute $\omega$ for the two-dimensional $p p$-wave $N_{2}$ in (4.9) obtained from the isometry $F_{2}$ in (4.7). Taking $k_{1}=t_{3}$ and $k_{2}=t_{4}$ in eqs. (4.3) and (4.4) and using eq. (6.1), it is straightforward that $\omega_{12}=\omega\left(k_{1}, k_{2}\right)=0$.

\section{Outlook}

In this paper we have found Lorentzian signature D-branes of all dimensions for the NappiWitten string background. We have achieved this by formulating the usual gluing condition $J_{+}=F J_{-}$for the corresponding WZW chiral currents $J_{+}$and $J_{-}$and by finding solutions for Lie algebra isometries $F$ that are not automorphisms, thus generalizing existing results. Our analysis shows that the methods used to obtain D-branes for Lie algebra automorphisms work very neatly for more general cases but require a careful formulation of integrability/involutivity. In particular, the occurrence of metrically degenerate D-branes and coordinate-dependent isometries $F(g)$ are solvable issues. We envisage various problems lying ahead. The most inmediate one is perhaps the study of the low-energy limit of the corresponding effective D-brane actions, somewhat in the way it is performed in ref. [7]. By doing so, we expect to learn about noncommutative field theory on curved Lorentzian D-branes and non-critical strings [4]. This may also provide a way to approach noncommutative solitons as bound states of strings [25].

\section{A Alternative computation of $\operatorname{rank}\left[\operatorname{Ad}_{g^{-1}}\left(-R_{ \pm}^{\Omega}\right)-1\right]$}

This appendix contains an alternative derivation to that given in the main text that the isometries $F=-R_{ \pm}^{\Omega}$ considered in subsections 4.1 and 4.2 do not define distributions for constant $R_{ \pm}^{\Omega}$. 
Since the adjoint group action $\operatorname{Ad}_{g^{-1}}$ defines for any group element $g$ an inner metricpreserving Lie algebra automorphism, $\operatorname{Ad}_{g^{-1}} R_{ \pm}^{\Omega}$ is the product of two automorphisms, hence an automorphism of the same type as $R_{ \pm}^{\Omega}$. In fact, eqs. (3.6) and (3.7) imply that

$$
\operatorname{Ad}_{g^{-1}} R_{ \pm}^{\Omega}(\rho, \phi, \theta)=R_{ \pm}^{\Omega}(\tilde{\rho}, \tilde{\phi}, \tilde{\theta}),
$$

where the parameters $\tilde{\rho}, \tilde{\phi}, \tilde{\theta}$ depend on $\rho, \phi, \theta$ and $g(x)$ through

$$
\begin{aligned}
\tilde{\phi} & =\phi \mp u \\
\tilde{\rho} \cos (\tilde{\theta} \mp \tilde{\phi}) & =\rho \cos (\theta \mp \phi+u) \pm x_{2} \\
\tilde{\rho} \sin (\tilde{\theta} \mp \tilde{\phi}) & =\rho \sin (\theta \mp \phi+u) \mp x_{1} .
\end{aligned}
$$

From eqs. (A.1) and (3.6) it follows that the rank of $\operatorname{Ad}_{g^{-1}} F-1$ is

$$
\operatorname{rank}\left(\operatorname{Ad}_{g^{-1}} R_{+}^{\Omega}+1\right)=\left\{\begin{array}{lll}
4 & \text { if } & \tilde{\phi} \neq(2 n+1) \pi \\
2 & \text { if } & \tilde{\phi}=(2 n+1) \pi
\end{array}\right.
$$

for $F=-R_{+}^{\Omega}$ and

$$
\operatorname{rank}\left(\operatorname{Ad}_{g^{-1}} R_{-}^{\Omega}+1\right)=\left\{\begin{array}{lll}
3 & \text { if } & \tilde{\rho} \sin \left(\tilde{\theta}+\frac{\tilde{\phi}}{2}\right) \neq 0 \\
1 & \text { if } & \tilde{\rho} \sin \left(\tilde{\theta}+\frac{\tilde{\phi}}{2}\right)=0
\end{array}\right.
$$

for $F=-R_{-}^{\Omega}$. We see that in both cases the rank of $\operatorname{Ad}_{g^{-1}} F-1$ is not constant over $G_{\mathrm{NW}}$. The fields $t_{a}=\left(\operatorname{Ad}_{g^{-1}} F-1\right) T_{a}$ hence do not provide a distribution on $G_{\mathrm{NW}}$.

\section{Acknowledgments}

The authors are grateful to MEC and UCM-BSCH, Spain for partial support through grants FPA2008-04906 and 910770(GR58/08). RHR acknowledges the Ramón y Cajal Program, Spain for support.

\section{References}

[1] C.-S. Chu and P.-M. Ho, Noncommutative open string and D-brane, Nucl. Phys. B 550 (1999) 151 [hep-th/9812219] [SPIRES].

[2] V. Schomerus, D-branes and deformation quantization, JHEP 06 (1999) 030 [hep-th/9903205] [SPIRES].

[3] N. Seiberg and E. Witten, String theory and noncommutative geometry, JHEP 09 (1999) 032 [hep-th/9908142] [SPIRES].

[4] N. Seiberg, L. Susskind and N. Toumbas, Strings in background electric field, space/time noncommutativity and a new noncritical string theory, JHEP 06 (2000) 021 [hep-th/0005040] [SPIRES].

[5] C.-S. Chu and P.-M. Ho, Noncommutative D-brane and open string in pp-wave background with B-field, Nucl. Phys. B 636 (2002) 141 [hep-th/0203186] [SPIRES]. 
[6] G. Horcajada and F. Ruiz Ruiz, Quantization of the open string on plane-wave limits of $d S_{n} \times S^{n}$ and non-commutativity outside branes, Nucl. Phys. B 799 (2008) 110 [arXiv:0711.2991] [SPIRES].

[7] A.Y. Alekseev, A. Recknagel and V. Schomerus, Non-commutative world-volume geometries: branes on $\mathrm{SU}(2)$ and fuzzy spheres, JHEP 09 (1999) 023 [hep-th/9908040] [SPIRES].

[8] A.Y. Alekseev, A. Recknagel and V. Schomerus, Brane dynamics in background fluxes and non-commutative geometry, JHEP 05 (2000) 010 [hep-th/0003187] [SPIRES].

[9] C. Klimčík and P. Ševera, Open strings and D-branes in WZNW models, Nucl. Phys. B 488 (1997) 653 [hep-th/9609112] [SPIRES].

[10] A.Y. Alekseev and V. Schomerus, D-branes in the WZW model, Phys. Rev. D 60 (1999) 061901 [hep-th/9812193] [SPIRES].

[11] S. Stanciu, D-branes in an AdS 3 background, JHEP 09 (1999) 028 [hep-th/9901122] [SPIRES].

[12] S. Stanciu, D-branes in group manifolds, JHEP 01 (2000) 025 [hep-th/9909163] [SPIRES].

[13] S. Stanciu, A note on D-branes in group manifolds: flux quantization and D0-charge, JHEP 10 (2000) 015 [hep-th/0006145] [SPIRES].

[14] J.M. Figueroa-O'Farrill and S. Stanciu, More D-branes in the Nappi-Witten background, JHEP 01 (2000) 024 [hep-th/9909164] [SPIRES].

[15] C. Bachas and M. Petropoulos, Anti-de-Sitter D-branes, JHEP 02 (2001) 025 [hep-th/0012234] [SPIRES].

[16] S. Ribault and V. Schomerus, Branes in the 2D black hole, JHEP 02 (2004) 019 [hep-th/0310024] [SPIRES].

[17] Y.-K.E. Cheung and L. Freidel, Inner brane: a D3-brane in the Nappi-Witten model from an inner group automorphism, Phys. Rev. D 79 (2009) 126007 [arXiv:0905.0540] [SPIRES].

[18] G. Horcajada and F.R. Ruiz, Geometric construction of D-branes in WZW models, arXiv: 1104.4722 [SPIRES].

[19] C.R. Nappi and E. Witten, A WZW model based on a nonsemisimple group, Phys. Rev. Lett. 71 (1993) 3751 [hep-th/9310112] [SPIRES].

[20] V. Schomerus, Lectures on branes in curved backgrounds, Class. Quant. Grav. 19 (2002) 5781 [hep-th/0209241] [SPIRES].

[21] V. Schomerus, Non-compact string backgrounds and non-rational CFT, Phys. Rept. 431 (2006) 39 [hep-th/0509155] [SPIRES].

[22] G. D'Appollonio and E. Kiritsis, D-branes and BCFT in Hpp-wave backgrounds, Nucl. Phys. B 712 (2005) 433 [hep-th/0410269] [SPIRES].

[23] E. Witten, Nonabelian bosonization in two dimensions, Commun. Math. Phys. 92 (1984) 455 [SPIRES].

[24] S. Stanciu and A.A. Tseytlin, D-branes in curved spacetime: Nappi-Witten background, JHEP 06 (1998) 010 [hep-th/9805006] [SPIRES].

[25] E. Witten, Bound states of strings and p-branes, Nucl. Phys. B 460 (1996) 335 [hep-th/9510135] [SPIRES]. 